\title{
SUCCESSFUL TREATMENT OF PULMONARY EMBOLISM AND ASSOCIATED UPPER EXTREMITY ARTERY THROMBOSIS USING INTRAVENOUS ALTEPLASE: A CASE REPORT
}

Kivanc Karaman*, Cihangir Celik*, Alten Oskay*, Hamit Hakan Armagan*, Onder Tomruk*

* Suleyman Demirel University, Faculty Of Medicine, Emergency Department, Isparta, TURKEY

\begin{abstract}
Background: Although thrombolytic administration is inevitable in the management of massive pulmonary embolism, contradictions exist in the treatment of sub-massive pulmonary embolism. There are drawbacks to thrombolytic treatment due to potential serious side effects such as cranial hemorrhage.

Case Report: An 84-year-old female patient was admitted to the emergency service because of pain and lack of a pulse in her right arm and \%61 oxygen saturation without any respiratory system complaints. A computerized tomography showed pulmonary embolism close to totality in the bilateral main pulmonary arteries and peripheral embolism in the right brachial artery. We considered a sub-massive pulmonary embolism in this patient with no hypotension despite bilateral pulmonary artery embolism, good general condition, but a dilated right ventriculi in transthoracic echocardiography. Since the PESI score was 115, it was decided that the patient was in the high-risk group. After administration of $100 \mathrm{mg}$ Alteplase as thrombolytic therapy, her oxygen saturation increased to 95\% in the room air and her complaint of right arm completely regressed. Furthermore, none of the possible complications from thrombolytic therapy, such as fatal bleeding, were observed in our patient.
\end{abstract}

Conclusion: In this article, it is emphasized that thrombolytic therapy is an appropriate choice for patients with sub-massive pulmonary embolism and acute arterial embolism.

Keywords: Emergency service, Peripheral arterial embolism, Pulmonary embolism, Thrombolytic therapy.

Received: 15 May 2018 Accepted:29 October 2018

\section{Introduction}

Pulmonary embolism (PE) is an important cause of morbidity and mortality in the emergency department. Patients with PE may be admitted to the hospital while asymptomatic or in cardiac arrest. In addition, there is a risk for recurrent $\mathrm{PE}$ and mortality in treated patients. Risk scoring systems for patients have been developed to assess these risks and standardize treatment. In particular, thrombolytic therapy is recommended in the high- and intermediate-risk groups, but there are different approaches to emergency treatment and long-term management.

Paradoxical embolism refers to the blockage of an artery due to a passage of a clot from a systemic vein to a systemic artery without passing through the lungs. Although rarely seen with peripheral embolisms, a concomitant PE causes serious morbidity and mortality.
In this case report, a successful outcome was obtained using alteplase treatment administered to an elderly female patient in the medium risk group with a high pulmonary embolism severity index score diagnosed with PE and peripheral arterial emboli. In addition, rivaroxaban treatment was chosen for the long-term management of the patient and successful results were obtained at 3 months follow-up.

\section{Case Report}

An 84-year-old female patient was admitted to the emergency service because of pain and lack of a pulse in her right arm. She had suffered right arm pain for approximately 12 hours. She had no respiratory system complaints. She denied any history of disease except hypertension and there was no history of any drug use. At admission, she was conscious and in generally good condition. Her blood pressure could not be measured in 
the right arm but was $120 / 70 \mathrm{~mm} / \mathrm{hg}$ in left arm, oxygen saturation was $61 \%$, and pulse was 85 beats per minute. Physical examination showed absence of any pulse in the right arm with a cold and pale appearance. No pathology was detected in other systemic examinations. Electrocardiography showed an inverted T wave in leads V1-4, suggestive of right ventricular (RV) loading (Picture 1). Chest X-ray films showed neither obvious congestion nor pneumonia. Examination of arterial blood gases revealed hypocapnic hypoxia ( $\mathrm{PaO} 232.4$ $\mathrm{mmHg}, \mathrm{PaCO} 235.6 \mathrm{mmHg}, \mathrm{pH} 7.36, \mathrm{HCO} 319.9 \mathrm{mmol} / \mathrm{L}$, SO2 55.5\%). In other laboratory tests, there was no pathologic finding other than detectable high sensitive troponin $\mathrm{T}(0.07 \mathrm{ng} / \mathrm{ml}$; normal range $0-0.01)$ and creatine kinase-MB (4.78 ng/ml; normal range 0-2.88). The $d$-dimer level of the patient could not be determined. Bedside transthoracic echocardiography (ECO) revealed moderate dilatation of the right ventricle, left ventricle ejection fraction of $\% 50$, pulmonary arterial pressure of $60 \mathrm{~mm} / \mathrm{hg}$, and a normal range of aortic root diameter. In order to evaluate $P E$, aortic aneurysm, and peripheral arterial embolism, it was decided to perform computerized tomography angiography for pulmonary artery and aorta. Although aortic dissection was not seen in the patient's computerized tomography, the flow was reoccurring because of the distal collateral filling after the flow was not observed in the right brachial artery. There was an embolism close to totality in the bilateral main pulmonary arteries and segmental arteries. Both pulmonary artery diameters were increased (Picture 2). We considered a sub-massive PE in this patient with no hypotension despite bilateral pulmonary artery embolism, good general condition, but a dilated RV in ECO. Since the pulmonary embolism severity index score was 115, it was decided that the patient was in the high-risk group. The patient was treated with $1000 \mathrm{U} / \mathrm{h}$ infusion following 5000 units intravenous bolus dose of heparin, and $100 \mathrm{mg}$ alteplase over 2 hours, which was recommended by the chest diseases and cardiovascular surgery departments. During the treatment, the patient had stable vital signs and had no consciousness or pupil changes. At the end of the thrombolytic therapy, the right arm pain completely regressed, peripheral pulses were detectable, and fingertip oxygen saturation at right arm increased to $95 \%$ in the room air. After a short follow-up period in the emergency department, the patient was admitted to the chest diseases clinic for further examination and treatment planning. In the chest diseases clinic, heparin infusion treatment was continued for 2 days. On the 2 nd day, oral rivaroxaban $2 \times 15 \mathrm{mg}$ was started and after discharge, the dose was reduced to $1 \times 20 \mathrm{mg}$. The patient returned to the outpatient clinic after three months for follow-up. She stated that she used her medication regularly and had no complaints over the 3 month period. No pathological findings were found during the physical examination.

Informed Consent: Written informed consent couldn't be obtained due to the impossibility of reaching the patient.

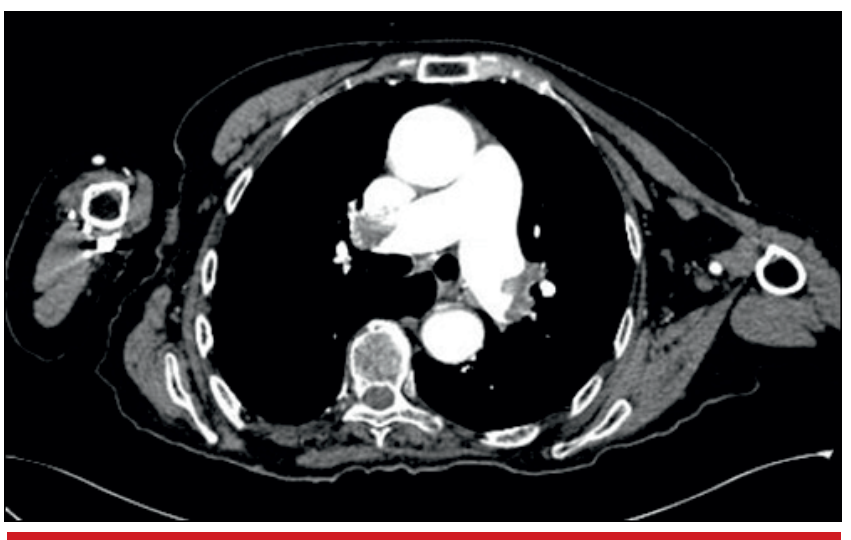

FIGURE 1. There was an embolism close to totality in the bilateral main pulmonary arteries and segmental arteries. Both pulmonary artery diameters were increased.

\section{Discussion}

Acute sub-massive PE is defined as normotensive patients with signs of RV dysfunction and/or myocardial injury and has a short-term mortality rate ranging from $3 \%$ to $15 \%$ (1). Thrombolytic use in massive PE is warranted, but patients with sub-massive $P E$ require case-by-case analysis with shared decision making. The risks, including major hemorrhage, and benefits, primarily improved long-term outcomes, should be considered $(2,3)$. An integrated multidisciplinary therapeutic approach in sub-massive PE is shown in Table 1 (1). 
TABLE 1. Proposed Algorithm Treatment For The Management Of Submassive Pulmonary Embolism (1).

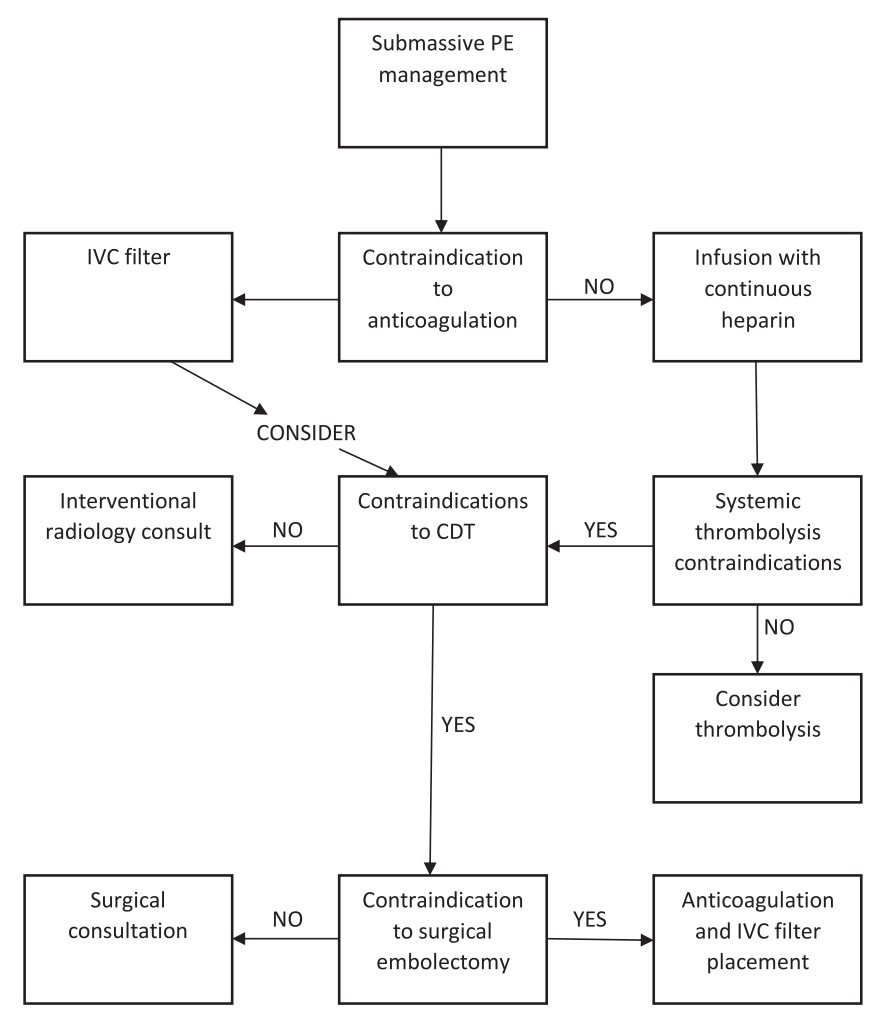

(CDT: Catheter Directed Therapy, IVC: Inferior Vena Cava)

In our case, we decided to administer intravenous heparin plus alteplase as a systemic thrombolytic. The patient's clinical symptoms decreased and oxygen saturation increased after the therapy. Furthermore, none of the possible complications from thrombolytic therapy, such as fatal bleeding, were observed in our patient. Konstantinides and colleagues reached conclusions that support our treatment in a multicenter study conducted in 2002. They concluded that alteplase plus heparin in sub-massive PE could improve clinical outcomes (4). In addition, they observed no fatal bleeding or cerebral bleeding in patients receiving heparin plus alteplase, similar to our case. In a prospective, placebo-controlled trial in which patients with similar symptoms to those presenting here were classified as 'moderately' $P E$, Sharifi et al. reported that $50 \mathrm{mg}$ alteplase treatment in addition to anticoagulant treatment was significantly better in pulmonary artery pressure reduction compared to anticoagulant therapy alone. In this study, no bleeding was observed in any patient treated with low dose thrombolytic therapy, but recurrent $P E$ and death were observed in 1 patient (5). In another study, Gao et al. concluded that thrombolytic therapy in patients with intermediate-risk PE is associated with lower all-cause mortality and recurrent $P E$ risk despite increased major and minor bleeding risks (6).

Guidelines recommend long-term oral anticoagulation with direct-acting oral anticoagulants or a vitamin $\mathrm{K}$ antagonist for the acute treatment of PE and secondary prevention of venous thromboembolism (7). Warfarin, historically the most widely used oral anticoagulant, was US Food and Drug Administration (FDA)-approved in 1954 , yet remains challenging to use in routine practice. Warfarin's narrow therapeutic index, high inter-patient variability, and significant drug-drug and drug-food interactions requires frequent laboratory monitoring and individualized dosing adjustments. Therefore, we prefer oral rivaroxaban for long-term anticoagulant therapy in our patients. Rivaroxaban is a direct oral factor Xa inhibitor and is US FDA approved to treat acute $P E$ and reduce the risk of recurrent venous thromboembolism without a requirement for bridging therapy or coagulation monitoring. Our patient was discharged on the 4th day of admission to the hospital and no recurrent $P E$, death, or bleeding was observed during the following 3 months. In the literature, as supporting our experience direct-acting oral anticoagulants were found to be as effective as Warfarin (8). A systematic review published by Kohn et al. in 2017 revealed that rivaroxaban is associated with a reduced hospital length of stay and costs versus warfarin, without increasing readmissions (9).

The patient was brought to the emergency room because of symptoms related to right arm circulation disorder and peripheral arterial embolism was detected in the right brachial artery. We concluded that the association of PE with peripheral arterial embolism is a paradoxical embolism, but we were not able to conduct detailed investigations because the clinical condition of the patient required urgent treatment. However, thrombolytic therapy successfully treated the PE as well as peripheral embolism. In a case reported by Işık et al. PE accompanying middle cerebral artery embolism was detected but they considered thrombolytic therapy was not appropriate because of the high risk of bleeding and the patient was died shortly after being diagnosed (10). Similar to our work, Zhang et al. reported a successful treatment with alteplase in 2 of 12 patients with PE and concomitant paradoxical embolism. In addition, warfarin was administered to these 2 patients for long- 
term treatment and long-term survival was reported (11). One of these patients had a bilateral popliteal artery embolism and the other had a descending aortal thrombus. In our patient, embolism was detected in the right brachial artery and a positive response to alteplase treatment was obtained. However, rivaroxaban has been the preferred anticoagulant for long-term treatment in our patient due to the reasons previously described.

The lack of detailed research in terms of paradoxical embolism in our patient has led to a limitation in the case presentation, although it did not affect our choice of treatment. In addition, control ECO may be useful to monitor changes in right ventricular function following thrombolytic therapy, but improvement in the patient's clinical symptoms was considered satisfactory and control ECO examination was not required.

\section{Conclusion}

Patients with sub-massive PE can be referred to emergency departments with different clinical presentations than classical symptoms. Although clinical findings are mild, thrombolytic therapy is especially safe and successful in high-risk sub-massive PE cases.

\section{References}

1. Teleb M, Porres-Aguilar M, Anaya-Ayala JE, Rodriguez-Castro $C$, Porres-Muñoz $M$, Mukherjee D. Potential role of systemic thrombolysis in acute submassive intermediate risk pulmonary embolism: review and future perspective. Ther Adv Cardiovasc Dis 2016; 10(2): 103-10.

2. Long B, Koyfman A. Current Controversies In Thrombolytic Use In Acute Pulmonary Embolism. The Journal of Emergency Medicine 2016; 51(1):37-44.

3. Baile'n MR, Cuadra JA, Aguayo De Hoyos E. Thrombolysis During Cardiopulmonary Resuscitation In Fulminant Pulmonary Embolism: A Review. Crit Care Med 2001; 29:2211-9.

4. Konstantinides S, Geibel A, Heusel G, Heinrich F, Kasper W. Heparin plus alteplase compared with heparin alone in patients with submassive pulmonary embolism. N Engl J Med 2002; 347: 1143-50.

5. Sharifi M, Bay C, Skrocki L, Rahimi F, Mehdipour M. Moderate pulmonary embolism treated with thrombolysis (from the MOPETT trial). Am J Cardiol 2013; 111:273-7.

6. Gao G, Yang P, Liu M, Din M, Liu G, Tong Y, et al. Thrombolysis for acute intermediaterisk pulmonary embolism: a meta-analysis. Thromb Res 2015; 136: 932-7.

7. Kearon C, Akl EA, Ornelas J, Blaivas A, Jimenez D, Bounameaux H, et al. Antithrombotic therapy for VTE disease: CHEST guideline and expert panel report. Chest 2016; 149: 315-52.

8. Van der Hulle T, Kooiman J, Den Exter PL, Dekkers OM, Klok FA, Huisman MV. Effectiveness and safety of novel oral anticoagulants as compared with vitamin $\mathrm{K}$ antagonists in the treatment of acute symptomatic venous thromboembolism: a systematic review and meta-analysis. J Thromb Haemost 2014; 12(3): 320-8.

9. Christine GK, Gregory JF, William FP, Phil SW, Christopher WB, Veronica $A$, et al. Association between rivaroxaban use and length of hospital stay, treatment costs and early outcomes in patients with pulmonary embolism:a systematic review of real-world studies. Current Medical Research and Opinion 2017; 33(9): 1697-703.

10. Işık GC, Çorbacıoğlu SK, Cevik Y. Watershed Infarct due to Massive Pulmonary Embolism: A Rare Diagnosis at the Emergency Department. JEMCR 2016; 7: 34-6.

11. Zhang HL, Liu ZH, Luo Q, Wang Y, Zhao ZH, Xiong CM. Paradoxical embolism: Experiences from a single center. Chronic Diseases and Translational Medicine 2017; 3: 123-8. 